\section{Topological perception: Holes in an experiment}

\section{JOHN M. RUBIN and NANCY KANWISHER Massachusetts Institute of Technology Cambridge, Massachusetts}

Chen (1982) reported three experiments to support his claim that a primitive function of visual systems is to extract the topological structure of objects (for example, connectedness, closedness, and number of holes). Chen's result was later synopsized for a large readership. ${ }^{1}$ Here we reinterpret one of Chen's three experiments and comment on the other two. After successfully replicating Chen's first result, we added a condition in which we controlled for luminous flux. Data from our control condition suggest that Chen's findings were due to a confounding of luminous flux with topological factors.

In Chen's first experiment, subjects were simultaneously shown a pair of figures and asked to respond "same" or "different." (The correct answer was always "different.") Chen found that, under near-threshold conditions, a pair of topologically distinct figures (diskannulus) was more discriminable than pairs of topologically identical but geometrically distinct ${ }^{2}$ figures (disksquare; disk-triangle). Chen concluded that the greater discriminability of the topologically distinct pair was due to the visual system's sensitivity to topological structure. ${ }^{3}$ However, the topological distinction was conflated with disparities in the luminous flux differences between the two figures of each pair. Indeed, the greater the flux difference, the better the discrimination. ${ }^{4}$

To claim a visual sensitivity to topological properties, a pair of figures of different topology but identical flux must be shown to be highly discriminable in an experimental situation similar to Chen's. In an attempt to replicate Chen's results, we added such a control pair (Figure 1a). The black area of the annulus is identical to the black area of the topologically distinct disk-with-two-holes. Two other stimulus pairs (Figures $1 \mathrm{c}$ and $1 \mathrm{~d}$ ) are similar to Chen's. A fourth case, annulus-horseshoe (Figure 1b), was added because it was topologically equivalent to the disk-annulus pair, yet had only a small (within-pair) difference in luminous flux. ${ }^{5}$

Following Chen, we used 5-msec tachistoscopic presentation of the stimuli. Our displays closely matched Chen's in visual angle subtended. ${ }^{6}$ With five naive subjects, we replicated Chen's finding that discriminability of the diskannulus pair was significantly greater than that of the disksquare $[\mathrm{t}(4)=2.9, \mathrm{p}<.025] .{ }^{7}$ However, the control

J.M.R. was supported in part by NSF Grant IST-8312240. N.K. was supported by NSF Grant BNS-8318156. The authors thank M. Potter and $W$. Richards for helpful comments. The authors' mailing address is: Department of Psychology (E10-104A), M.I.T., 79 Amherst St., Cambridge, MA 02139.

\begin{tabular}{|c|c|c|c|c|}
\hline & $\mathbf{A}$ & B & C & D \\
\hline STIMULUS & & & & \\
\hline $\begin{array}{l}\text { TOPOLOGICAL } \\
\text { DIFFERENCE }\end{array}$ & YES & YES & No & YES \\
\hline $\begin{array}{l}\text { BLACK AREA } \\
\text { DIFFERENCE }\end{array}$ & $0 \mathrm{~mm}^{2}$ & $92 \mathrm{~mm}^{2}$ & $96 \mathrm{~mm}^{2}$ & $254 \mathrm{~mm}^{2}$ \\
\hline$\%$ "DIFFERENT" & 33.6 & 35.1 & 44.4 & 63.5 \\
\hline
\end{tabular}

Figure 1. Experimental stimuli and mean percent "different" (correct) in the discrimination task $(n=5)$. Also shown are the difference in black area for each figure pair (which is roughly proportional to flux difference) and which pairs are topologically distinct.

stimulus (Figure 1a) was not significantly more discriminable than disk-square. Thus, subjects failed to respond to a topological distinction between two stimuli balanced for luminous flux. Furthermore, the annulushorseshoe pair, having the same topological difference as the disk-annulus pair, yet being more closely balanced in flux, failed to be significantly more discriminable than the disk-square pair. Thus, we found that discriminability was attributable to (within-pair) differences in luminous flux but not topology.

It has long been known that the primate visual system has a primitive capacity-one that survives even bilateral removal of visual cortex - to detect differences in luminous flux (Klüver, 1941). We believe it is that capacity that best accounts for Chen's findings.

In Chen's second and third experiments, respectively, he claimed that the discriminability of line segments can be enhanced by being presented as part of a figure having the topological properties of (1) closedness and connectedness, or (2) inside versus outside. However, Chen did not test the advantage of geometrically similar contexts lacking these topological properties, and has thus failed to distinguish between the mere presence of context and a topological property of context as the factor influencing detection. The former provides the more parsimonious account, particularly since the determination of topological properties is not likely to be primitive for any kind of computational system (Minsky \& Papert, 1969; Ullman, in press).

How the visual system responds to aspects of topological structure is an interesting question. Answering that question will be challenging since topological characteristics of figures are necessarily confounded with metrical properties such as area and perimeter length.

\section{REFERENCES}

Chen, L. (1982). Topological structure in visual perception. Science, 218, 699-700.

KLÜVER, H. (1941). Visual functions after removal of the occipital lobes. Journal of Psychology, 11, 23-45.

Minsky, M. L., \& PAPERT, S. (1969). Perceptrons: An introduction to computational geometry. Cambridge, MA: M.I.T. Press. 
ULLMAN, S. (in press). Visual routines. In W. Richards \& S. Ullman (Eds.), Image Understanding 1985. Norwood, NJ: Ablex. (Also to appear in Cognition.)

\section{NOTES}

1. "Science and the Citizen," Scientific American, 1983, 248(2), 83.

2. Geometry describes measurable properties of figures such as size and shape, whereas topology concerns properties that are invariant over smooth deformations of shape and changes in size.

3. Chen sees his argument strengthened by the finding that the difference in discriminability between his two same-topology pairs does not reach significance $[t(5)=1.37, p<.20]$. However, the lack of a significant difference is hardly evidence for equality. Instead, his data indicate a difference in discriminability between the same-topology pairs, with roughly $80 \%$ probability that this difference is not due to chance.

4. Chen's figures were solid black on a white background. The black areas of the figures can be determined from Chen's description of the stimuli: disk, $804 \mathrm{~mm}^{2}$; square, $1,024 \mathrm{~mm}^{2}$; triangle, $621 \mathrm{~mm}^{2}$; annulus, $550 \mathrm{~mm}^{2}$. Figures with larger black areas obviously give rise to less luminous flux. Consider next the difference in black areas of the stimulus pairs: disk-triangle, $183 \mathrm{~mm}^{2}$; disk-square, $220 \mathrm{~mm}^{2}$; diskannulus, $254 \mathrm{~mm}^{2}$. Chen reports that the disk-triangle pair (having the smallest difference in luminous flux between the members of the pair) was the least discriminable pair. The disk-annulus stimulus (having the largest flux difference) was the most discriminable pair.

5. The square of Figure 1d has sides of $30 \mathrm{~mm}$. The black diskused to construct all of the circular figures in our experiment-has a diameter of $32 \mathrm{~mm}$. The inner radius of the annulus is $18 \mathrm{~mm}$. The horseshoe of Figure $1 \mathrm{~b}$ is identical to the annulus except that a $60^{\circ}$ sector is removed. The two holes of the disk-with-two-holes of Figure la are semicircles of diameter $18 \mathrm{~mm}$. The difference in the black areas of each figure pair are shown in Figure 1.

6. Our figures have identical dimensions to Chen's except for our square, whose sides are $2 \mathrm{~mm}$ shorter. Chen and we used a tachistoscope of length $90 \mathrm{~cm}$.

7. Subjects were run on 64 intermixed trials (each stimulus pair of Figure 1 was thus shown 16 times). The subjects were asked to respond "same" or "different," although all pairs were in fact different. Thus, percent "different" was percent correct and a measure of discriminability of each pair. For each subject, a percent "different" score was calculated for each of the four stimulus conditions (stimulus pairs). These scores were used for $t$ tests between conditions across subjects.

Six naive subjects were run. One subject's data were deemed inappropriate as this subject failed to replicate Chen's findings. These data showed a disk-annulus disadvantage of over $60 \%$ as compared with the disk-square. 